Article

\title{
A Paper-based Immunofluorescent Device for the Detection of SARS-CoV-2 Humanized Antibody
}

\author{
Surasak Kasetsirikul 1,2, Muhammad Umer ${ }^{2}$, Narshone Soda ${ }^{2,3}$, Kamalalayam Rajan Sreejith ${ }^{2}$, \\ Muhammad J. A. Shiddiky 2,3,", and Nam-Trung Nguyen 2,* \\ 1 School of Engineering and Build Environment (EBE), Griffith University, Nathan Campus, QLD 4222, \\ Australia. \\ 2 Queensland Micro-and Nanotechnology Centre (QMNC), Griffith University, Nathan Campus, QLD 4111, \\ Australia \\ 3 School of Environment and Science (ESC), Griffith University, Nathan Campus, QLD 4111, Australia \\ * Correspondence: : m.shiddiky@griffith.edu.au, nam-trung.nguyen@griffith.edu.au
}

\begin{abstract}
We report on an immunofluorescent paper-based assay for the detection of severe acute respiratory symptom coronavirus 2 (SARS-CoV-2) humanized antibody. The paper-based device was fabricated by using lamination technique for easy and optimized handling. Our approach utilises a two-step strategy that involves (i) initial coating of the paper-electrode with recombinant SARS-CoV-2 nucleocapsid antigen to capture the target SARS-CoV-2 specific antibodies, and (ii) subsequent detection of SARS-CoV-2 antibodies using fluorophore-conjugated IgG antibody. The fluorescence readout was observed with fluorescence microscopy. The images were processed and quantified using a MATLAB program. The assay can selectively detect SARS-CoV-2 humanized antibodies spiked in PBS and healthy human serum samples with the relative standard deviation of approximately $6.4 \%$ (for $\mathrm{n}=3$ ). It has broad dynamic ranges $(1 \mathrm{ng}$ to $50 \mathrm{ng} / \mu \mathrm{L}$ in PBS and 5 to 100 $\mathrm{ng} / \mu \mathrm{L}$ in human serum samples) for SARS-CoV-2 humanized antibodies with the detection limits of $2 \mathrm{ng} / \mu \mathrm{L}(0.025 \mathrm{IU} / \mathrm{mL})$ and $10 \mathrm{ng} / \mu \mathrm{L}(0.125 \mathrm{IU} / \mathrm{mL})$ in PBS and human serum samples, respectively. We believe that our assay has the potential to be used as a simple, rapid, and inexpensive paper-based diagnostic device with a portable fluorescent reader to provide point-of-care diagnosis. This assay can be used for rapid examination of a large batch of samples toward clinical screening of SARS-CoV-2 specific antibodies as a confirmed infected active case or to evaluate the immune response to a SARS-CoV-2 vaccine.
\end{abstract}

Keywords: SARS-CoV-2 detection, Immunofluorescence, Paper-based diagnostic device

\section{Introduction}

World Health Organization (WHO) declared a public health emergency of international concern in January 2020 resulting from an increase in anomalous pneumonia patients in Wuhan, China and nearby areas.[1,2] It was confirmed by genome sequence analysis that the symptom originated from viral infection by a member of Coronaviridae family closely related to Severe Acute Respiratory Symptom Coronavirus (SARS-CoV) and Middle East Respiratory Symptom Coronavirus (MERS-CoV).[3, 4] Severe Acute Respiratory Symptom Coronavirus-2 (SARS-CoV-2), 2019 novel coronavirus (2019-nCoV) or COVID-19 were named by International Virus Classification Commission in March 2020. [5] SARS-CoV-2 is a single stranded and positive-sense RNA virus with a genome size of $\sim 30 \mathrm{~kb}$, which has about $89 \%$ similarity with SARS-CoV. [5, 6] SARS-CoV-2 has spread throughout the globe causing 171.0 million confirmed cases of infection and 3.5 million confirmed deaths, reported on 3 June 2021 by European Centre for Disease Prevention and Control (ECDC).

Infected patients develop a broad range of symptoms from mild flu-like symptoms to severe pneumonia leading to death. [7] Most importantly, some patients have an ability to spread the 
virus in the early stage without developing any symptoms, which makes the situation more difficult and out-of-control.[8] With this regard, many governments have made a decision to lockdown their countries, tightening migration regulations such as state quarantine or medical certificate for travelling, implemented social distancing procedure to minimize the spread of the disease.[6, 9, 10] Consequently, diagnostic test for detecting SARS-CoV-2 patients is urgently needed to identify and separate patients from the community. To date, the most accurate and sensitive diagnostic method deemed as gold standard for SARS-CoV-2 detection is reverse transcription polymerase chain reaction (RT-PCR) because this method directly detects viral RNAs in the sample from oropharyngeal and deep nasal swabs.[11] Nonetheless, well-trained staff and high-level biosafety facility are required to collect samples and perform the diagnostic test.[12] Another promising diagnostic method for SARS-CoV-2 detection is serological testing, which employs antigen and antibody interaction. Some proteins enriched on viral membrane could be an alternative for SARS-CoV-2 detection, or antibodies could be developed resulting from host immune response.[6] In case of immune response, antibodies in the host which are immunoglobin $G(\operatorname{IgG})$ and immunoglobin $\mathrm{M}(\mathrm{IgM})$ play an important role in developing a specific response against invading pathogen. Therefore, some serological assays are devised to detect specific SARS-CoV-2 antibody in human blood samples. The number of antibodies reaching detectable range could take from 5 day to 2 weeks after the infection because the immune response is dependent on the status of immune system of each patient.[11, 13] Thus, serological test is not appropriate for early diagnosis for timely identifying and isolating infected patients from the community. Novel and alternative method including lateral flow immunoassay (LFIA) or rapid diagnostic tests (RDTs) for SARS-CoV-2 diagnosis have been reported in several review articles.[14, 15]

Nevertheless, each diagnostic method has its own advantages and disadvantages. Different diagnostic tests should be performed to interpret the result accurately. Moreover, in the current stage of vaccine development, serological assay for determination of post-vaccination SARS-CoV-2 antibody in patients is urgently needed to evaluate the vaccine efficacy in a large number of human trial sample.[16, 17] In this study, we developed a relatively sensitive, rapid and inexpensive platform using Immunofluorescent paper-based ELISA for SARS-CoV-2 antibody detection. This paper-based assay employed antigen-antibody interaction in accordance with fluorophore for signal emission. The target antibody, SARS-CoV-2 humanized antibody in this case, was captured by coating recombinant SARS-CoV-2 nucleocapsid antigen on the paper. Next, secondary antibody conjugated with fluorophore was loaded to form immunocomplex and emit fluorescent signal after being excited by a light source with a specific wavelength. The assay can be completed within 15 min, which is more rapid than conventional ELISA (1-2 h) and our previous study on colorimetric paper-based ELISA (30 min).[18] Immunofluorescent paper-based ELISA is a promising platform for diagnostic assay particularly for limited-resource environment.[19] In addition, this assay also successfully worked with human serum sample, showing that our assay is feasible for complex biological samples.

\section{Materials and Methods}

\subsection{Reagents and Instrumentation}

Recombinant SARS-CoV-2 nucleocapsid (MBS355892, MyBioSource, USA), SARS-CoV-2 Nucleocapsid humanized antibody (MBS355887, MyBioSource, USA) and Rabbit anti-human IgG (ab97156, Abcam, UK) conjugated with fluorophore by fluorescent conjugation kits (ab236553, Abcam, UK) were used for immunofluorescent assay. 10\% Bovine Serum Albumin (BSA, A1595, Sigma Aldrich, USA) was used as a blocking buffer. Phosphate Buffer Saline (PBS) was prepared from a tablet (10388739, Fisher Scientific, USA) by dissolving it in DI water (Milli-Q, Merck, USA). Tween-20 detergent (655204), EMD, Millipore, USA) was used as a surfactant for PBST containing $0.05 \%$ Tween-20. For complex biological sample, human serum (H4522, Sigma Aldrich) was used as a mock human sample. For image acquisition system, fluorescent microscope (Nikon Eclipse Ti-2, Nikon, Japan) was used to capture image with excitation wavelength at $488 \mathrm{~nm}$ from light source 
(pE-4000, CoolLED, UK) and exposure time at 300 milliseconds. Then, the image was observed and recorded by an imaging software (NIS-Elements AR 5.00.00, Nikon, Japan). Image processing and quantification were done with a customized analysis program (MATLAB R2018b, The MathWorks Inc, USA).

\subsection{Paper-based device fabrication and assay preparation}

Paper-based device fabrication and assay preparation were adapted from our previous study (Figure 1).[18] Briefly, Whatman chromatography filter paper (Whatman, UK) was cut into 5-mm diameter and aligned at the centre between two laminated films with a 4-mm diameter hole. The paper in laminated films (PLF) was fed into a laminator at $120^{\circ} \mathrm{C}$ at a speed of $10 \mathrm{~mm} / \mathrm{s}$. Consequently, $5 \mu \mathrm{L}$ of $0.2 \mathrm{mg} / \mathrm{mL}$ recombinant SARS-CoV-2 nucleocapsid in $2 \%$ BSA was dropped on the PLF and incubated in humidified box for $10 \mathrm{~min}$. Subsequently, PLF was dried in the incubator at $37^{\circ} \mathrm{C}$ for $10 \mathrm{~min} .10 \mu \mathrm{L}$ of PBS was added twice on PLF surface and removed by putting blotting paper underneath to remove unbound antigen. After washing, PLF was dried for 10 min in the incubator at $37^{\circ} \mathrm{C}$ and kept in a petri dish at room temperature for further use.

\subsection{SARS-CoV-2 humanized antibody immunofluorescent detection assay}

The assay used in this study is an immunofluorescent detection. A sample volume of $5 \mu \mathrm{L}$ was added into the prepared PLF device and incubated for $10 \mathrm{~min}$. Then, the device was washed with 10 $\mu \mathrm{L}$ of PBST thrice to remove unbound molecules. Subsequently, $3 \mu \mathrm{L}$ of $10 \mathrm{ng} / \mu \mathrm{L}$ rabbit anti-human IgG conjugated with AlexaFlour488 was incubated for $3 \mathrm{~min}$ to bind to the SARS-CoV-2 antibodies and form an immunocomplex. Next, the paper was washed with $10 \mu \mathrm{L}$ PBST thrice. For a better image quality, the device was dried for $10 \mathrm{~min}$ to remove excess water in the paper which may reflect or reduce the fluorescent signal. For negative sample, only PBS or non-spiked healthy human serum were used for background signal as some secondary antibody tagged with fluorophore may not be completely removed from the device.

\subsection{Fluorescent readout and data processing}

After adding secondary antibody conjugated with fluorophore, the paper device was observed, and images were captured under a fluorescent microscope. Qualitative data was observed as a bright green colour under excitation at a wavelength of $488 \mathrm{~nm}$. All images were processed and quantified with MATLAB. Briefly, the image was individually imported by MATLAB. The area of

(a)

(b)
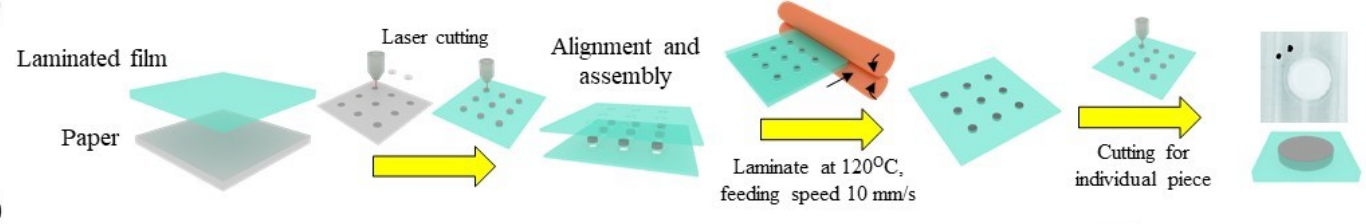

Coating Recombinant SARS-CoV- 2 in $2 \%$ BSA

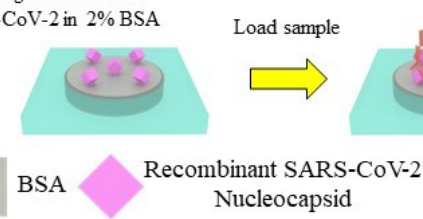

Loading detecting Anti-human IgG-AlexaFluor 488
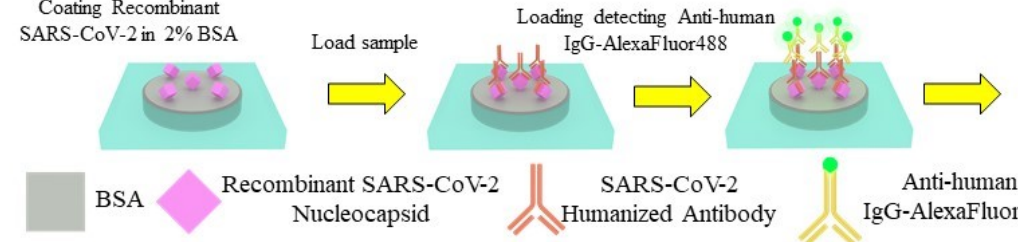

Nucleocapsid
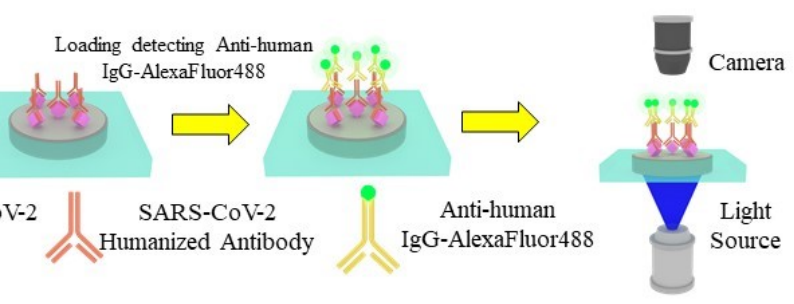

Positive

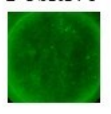

Negative

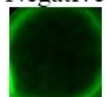

Figure 1 (a) Schematic diagram of paper fabrication and (b) assay design and detection process for SARS-CoV-2 humanized antibody detection assay. The paper was manufactured by lamination for easy handling and prepared with recombinant SARS-CoV-2 antigen to capture SARS-CoV-2 humanized antibody in the sample. Subsequently, the paper was observed under fluorescent microscope to validate the detection assay. 
interest was cropped as a square box from the centre of circular working area, and the original RGB colour image was subsequently split into R, G and B channel as a grayscale image. However, the fluorescent image emitted fluorescent signal at wavelength $525 \mathrm{~nm}$ which is green. Therefore, only the mean grey value $(G)$ from the green channel was used to quantify the fluorescent signal. To confirm that the colour originates from the immunofluorescent assay, the value reported in this study was subtracted by the background signal from negative samples as:

$$
\text { Gvalue }=G-G \text { neg }
$$

where $G$ is the mean grey value of the sample of interest, and Gneg is the mean grey value from negative sample. All experiments were individually performed with a sample number of at least $n=$ 3. The error bar is determined by standard deviation of repetitive experiments.

\section{Result and Discussion}

Figure 1 shows the assay protocol. SARS-CoV-2 humanized antibody was detected using immunofluorescence assay. The target antibody (humanized antibody SARS-CoV-2) was captured by recombinant SARS-CoV-2 antigen immobilized on the paper and formed an immunocomplex with secondary antibody conjugated with fluorophore. When excited at a wavelength of $488 \mathrm{~nm}$, the fluorescent tag emits bright green light and the image was taken. The intensity of the bright green colour is proportional to the concentration of SARS-CoV-2 humanized antibody in the sample. To demonstrate the specificity and validity of the assay, we performed the assay with a sample consisting of SARS-CoV-2 humanized antibody at $20 \mathrm{ng} / \mu \mathrm{L}$ and $50 \mathrm{ng} / \mu \mathrm{L}$ (Positive control), while the sample without SARS-CoV-2 humanized antibody included anti-human CA125 (wrong target), which is non-specific to recombinant SARS-CoV-2 antigen (Figure 2). Moreover, for the negative control experiment, the absence of secondary antibody conjugated with fluorophore (no secondary antibody) led to a low fluorescence intensity. The other negative control experiment, where no recombinant SARS-CoV-2 antigen (No recombinant SARS-CoV-2) was immobilized on the paper and wrong antigen with anti-human CA125 (wrong coating antibody), also shows low intensity. These experiments suggest that our assay relies on the presence of SARS-CoV-2 humanized antibody and recombinant SARS-CoV-2 antigen. The data indicates that positive control showed relatively higher response than non-specific samples, suggesting that our assay is suitable for detecting specific targets even if it is affected by high background signal. It is noted that the background signals for No recombinant SARS-CoV-2 and Wrong Coating Antibody cases were higher than the case of No Secondary Antibody and Wrong Target. This could be the residue of
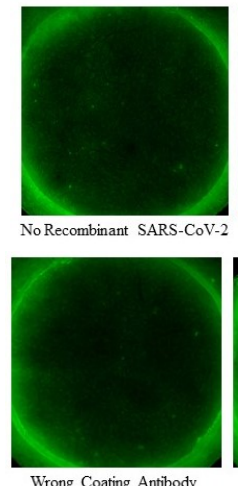

Wrong Coating Antbody
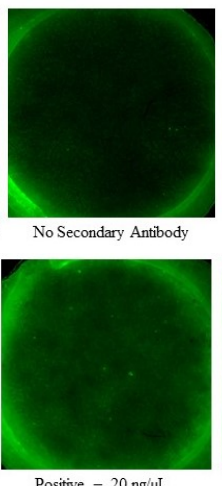

Positive $-20 \mathrm{ng} / \mathrm{LL}$

(a)

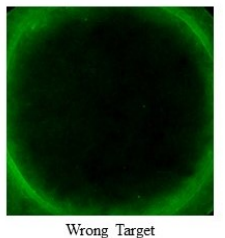

Wrong Target

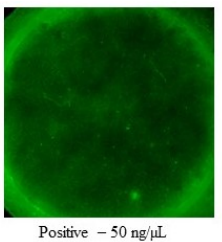

Positive $-50 \mathrm{ng} / \mathrm{LL}$

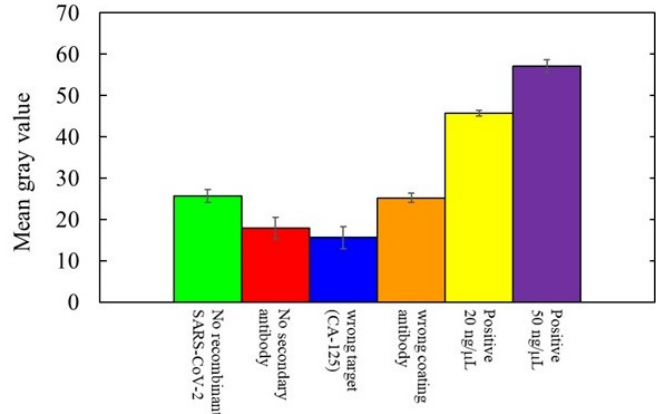

(b)

Figure 2 (a) Schematic diagram of paper fabrication and (b) assay design and detection process for SARS-CoV-2 humanized antibody detection assay. The paper was manufactured by lamination for easy handling and prepared with recombinant SARS-CoV-2 antigen to capture SARS-CoV-2 humanized antibody in the sample. Subsequently, the paper was observed under fluorescent microscope to validate the detection assay. 
secondary antibody tagged with fluorophore left on the paper. Many previous paper-based immunofluorescent assay studies also found the residue of antibody with fluorophore molecules in a negative sample. [18-20] An alternative way to minimize the background signal is to use black paper. [20] For clinical sample analysis such as human serum, plasma, etc., the background signal could be decreased by diluting the sample to minimize complex substance resulting in high background signal.

Conventional ELISA for serological tests available in the market are considered as a qualitative assay (Yes/No assay) based on result interpretation from different laboratories. Nevertheless, the assay in this study evaluated various starting antibody concentrations in the sample. Fluorescent readout can be performed by the software which can easily integrated into a low-cost, and portable reader for point-of-care use. Known concentrations of SARS-CoV-2 humanized antibody were prepared in PBS $(0.5,1,2,5,10,20,50$, and $100 \mathrm{ng} / \mu \mathrm{L})$ to determine the sensitivity of the assay. Figure $3 a$ illustrates the series of image readouts of the bright green intensity and antibody concentration. Following the sequential images of different antibody concentrations, the emitted fluorescence on the paper was processed and quantified. The increasing trend of the Gvalue agrees well with the increasing of SARS-CoV-2 humanized antibody concentration in the range from 0.5 to $100 \mathrm{ng} / \mu \mathrm{L}$. The increasing fluorescent readout results from a higher concentration of SARS-CoV-2 humanized antibody because these increasing number of antibodies can capture anti--human IgG antibody conjugated with fluorophore leading to a higher fluorescent signal detection. The linear equation for the assay was fitted to be $y=0.6333 x+5.9884$, with $\mathrm{R} 2=0.8904$ for SARS-CoV-2 humanized antibody concentration from 1 to $50 \mathrm{ng} / \mu \mathrm{L}$ in the linear region (the photo inset in Figure 3c). The limited of detection (LOD) was indicated by the linear equation and the Gvalue of the positive sample is approximately twice than that of the background signal. These data suggest that LOD of this assay is $2 \mathrm{ng} / \mu \mathrm{L}$ or $0.025 \mathrm{IU} / \mathrm{mL}$. Interestingly, our LOD is significantly lower than that of some conventional ELISA IgG kits in the market $(5 \mathrm{IU} / \mathrm{mL})$ and that of previous paper-based ELISA

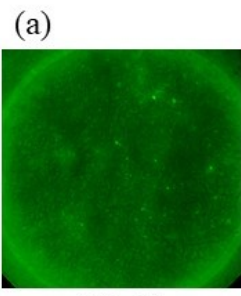

$100 \mathrm{ng} / \mu \mathrm{L}$

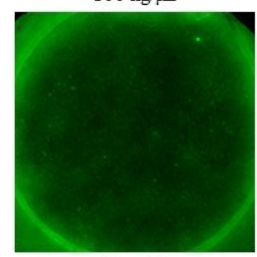

$10 \mathrm{ng} / \mu \mathrm{L}$

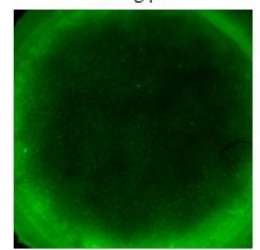

$1 \mathrm{ng} / \mu \mathrm{L}$

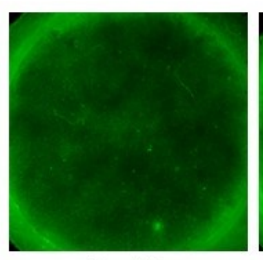

$50 \mathrm{ng} / \mu \mathrm{L}$

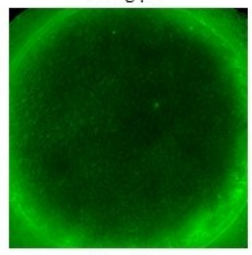

$5 \mathrm{ng} / \mu \mathrm{L}$

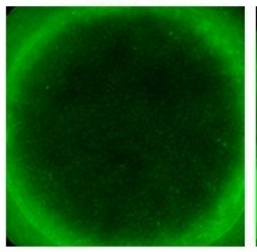

$0.5 \mathrm{ng} / \mu \mathrm{L}$

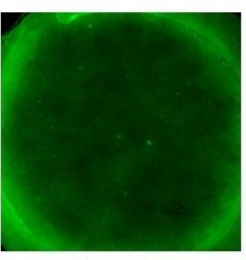

$20 \mathrm{ng} / \mu \mathrm{L}$

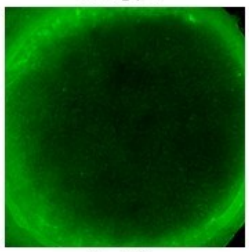

$2 \mathrm{ng} / \mu \mathrm{L}$

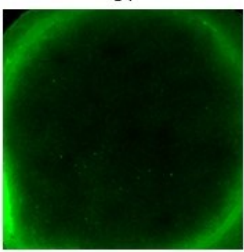

Negative Control (b)

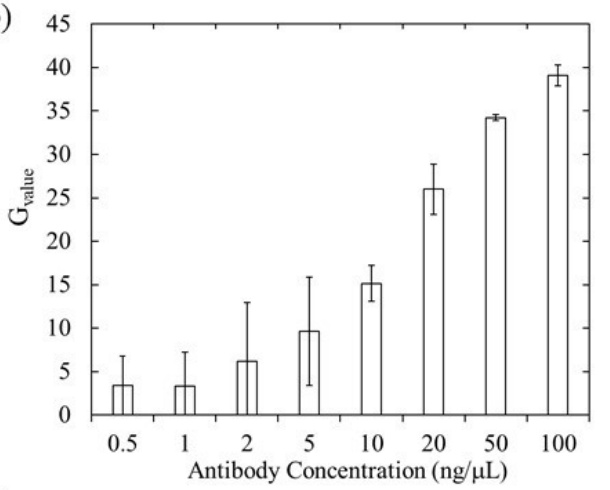

(c)

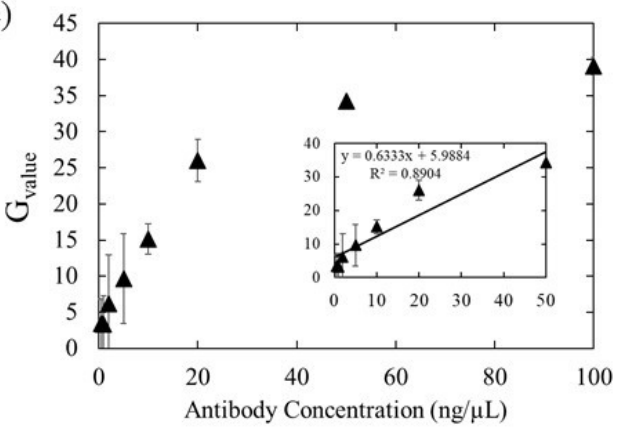

Figure 3 Sensitivity of the assay (a) Fluorescent images of known SARS-CoV-2 humanized antibody concentration from 0.5 to $100 \mathrm{ng} / \mu \mathrm{L}$ (b) The bar diagram for Gvalue observed from known SARS-CoV-2 humanized antibody concentration from 0.5 to $100 \mathrm{ng} / \mu \mathrm{L}$ (c) The relation of Gvalue and SARS-CoV-2 humanized antibody concentration. The inset shows the linear calibration plot from 1 to $50 \mathrm{ng} / \mu \mathrm{L}$. 


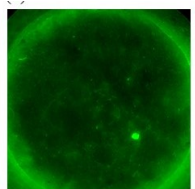

Positive\#1

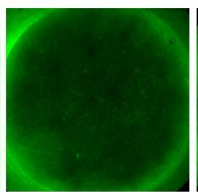

Positive\#2

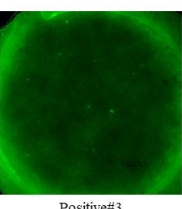

(a)

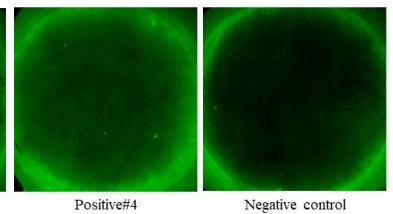

Negative control

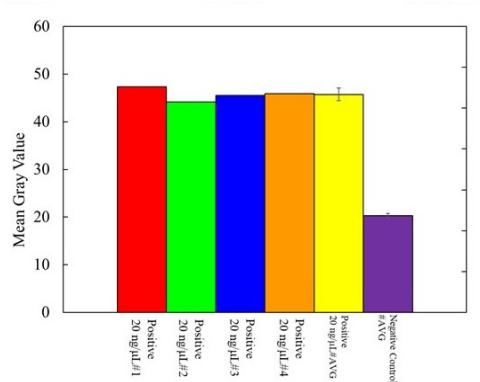

(b)

Figure 4 Repeatability of the assay (a) Fluorescent images obtained from independent assay experiment (Positive100ng\#1, \#2, \#3, and \#4) with 20 ng/ $\mu$ L SARS-CoV-2 humanized antibody (b) The bar diagram for mean grey value observed from independent experiment comparing to control one.

(0.012 IU/mL).[18] Moreover, we also investigated the repeatability of our assay. Figure 4a shows that fluorescent readout from individual experiment at $20 \mathrm{ng} / \mu \mathrm{L}$ SARS-CoV-2 humanized antibody was quantified and provided consistent data. The quantification data suggests that relative standard deviation (\%RSD) of our assay is approximately $6.4 \%$ for sample $n=3$ (Figure $4 \mathrm{~b}$ ). The repeatability and \%RSD of this assay are also lower than that of conventional ELISA IgG kit assay and previous paper-based ELISA (15\%-20\% and 10\% respectively)

To date, there is no study on fluorescent paper-based immunoassay for SARS-CoV-2 humanized antibody detection. However, there are a few reports on paper-based fluorescent ELISA working with other proteins and antibody detection. The linear range from our study is similar or better (100-fold concentration) than those of the reported values from paper-based fluorescent ELISA: Carcinoembryonic antigen (CEA) $(0.1-80 \mathrm{ng} / \mathrm{mL})$, Alpha-fetoprotein (AFP) $(0.1-80 \mathrm{ng} / \mathrm{mL})$, CA199 (0.1 - $80 \mathrm{U} / \mathrm{mL})$, Procalcitonin (PCT) (0.3 - $200 \mathrm{ng} / \mathrm{mL})$, C-reactive protein (CRP) (50-250 $\mu \mathrm{g} / \mathrm{mL})$, and aflatoxin $\mathrm{B}_{1}(5-150 \mathrm{pg} / \mathrm{mL})$. [20-22] Recently, we reported a colorimetric paper-based ELISA for SARS-CoV-2 humanized antibody detection. The linear working range of antibody concentration (1 to $50 \mathrm{ng} / \mu \mathrm{L})$ is similar to the fluorescent assay in this paper $(0.5$ to $50 \mathrm{ng} / \mu \mathrm{L})$.[18] As protein-to-protein variation, different proteins and antibodies could be detected in various ranges of concentration.[23] Thus, antibody detection on paper-based fluorescent ELISA assay is required to be optimized for working detection ranges.

We also studied the feasibility of our assay for more complex biological samples by spiking target antibodies in healthy human serum samples. Briefly, SARS-CoV-2 humanized antibody was spiked into the human serum with ratios of 1:1, 1:10 and 1:100 between human serum and SARS-CoV-2 humanized antibody solution. Figure 5a depicts the readout of the fluorescent intensity of SARS-CoV-2 antibody concentration of $50 \mathrm{ng} / \mu \mathrm{L}$. The bar diagram depicted Gvalue from different
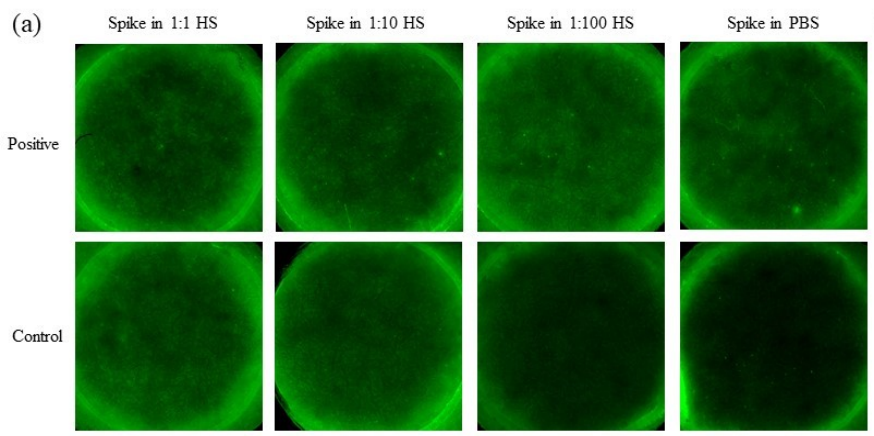

(b)

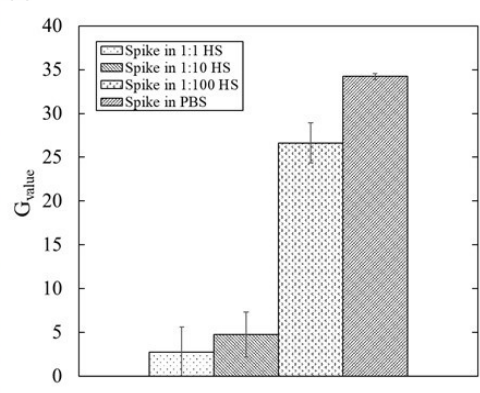

Figure 5 Spike sample analysis (a) Fluorescent images of known SARS-CoV-2 humanized antibody concentration at $50 \mathrm{ng} / \mu \mathrm{L}$ spiked in human serum ratio of 1:1, 1:10 and 1:100 (b) The bar diagram for Gvalue observed from known SARS-CoV-2 humanized antibody concentration at $50 \mathrm{ng} / \mu \mathrm{L}$ spiked in human serum ratio of 1:1, 1:10 and 1:100 
ratios of human serum in the presence of SARS-CoV-2 antibody. The more concentration of human serum in the sample, the less Gvalue could be observed. This finding could be explicated that healthy human serum may contain several biomolecules, which could bind with antibody conjugated with fluorophore leading to background signal due to fluorescent residue left on the paper.[18] Therefore, diluted human serum could reduce the background signal. This data suggested that human serum sample should be diluted at ratio 1:100 to maximize fluorescent signal. It is worth noting that the Gvalue from SARS-CoV-2 antibody spiked in PBS is higher than that of SARS-CoV-2 antibody spiked in human serum ratio of 1:100 dilution as impurity in human serum may increase background signal. In addition, known SARS-CoV-2 humanized antibody concentrations were prepared in human serum with ratio 1:100 dilution sample $(5,10,20,50$, and $100 \mathrm{ng} / \mu \mathrm{L})$ Figure 6a depicts the bright green intensity versus the increasing antibody concentration. An increasing trend of Gvalue corresponds to the increase of SARS-CoV-2 humanized antibody concentration from 5 to $100 \mathrm{ng} / \mu \mathrm{L}$ (Figure 6b) The linear equation of fluorescent ELISA assay for SARS-CoV-2 humanized antibody concentration spiked in human serum with ratio of 1:100 dilution was fitted to be $\mathrm{y}=0.3471 \mathrm{x}+$ 5.6771, with R2 = 0.962 with the data set of SARS-CoV-2 antibody concentration from 5 to $100 \mathrm{ng} / \mu \mathrm{L}$ (Figure 6c) These data advise that LOD of the assay for SARS-CoV-2 antibody spiked in human serum with ratio of $1: 100$ dilution is $10 \mathrm{ng} / \mu \mathrm{L}$ or $0.125 \mathrm{IU} / \mathrm{mL}$. Our LOD is also significantly lower than that of some conventional ELISA IgG kits in the market $(0.125 \mathrm{vs} 5 \mathrm{IU} / \mathrm{mL})$, which the conventional assay also diluted the human sample with ratio of 1:100.[18]

\section{Conclusion}

We have developed a paper-based fluorescent ELISA assay for SARS-CoV-2. We performed the immunofluorescence for SARS-CoV-2 humanized antibody detection in two types of sample: PBS for proof-of-concept assay and human serum for demonstrating application in real human samples. Experimental data suggest that this assay can detect SARS-CoV-2 humanized antibody at $2 \mathrm{ng} / \mu \mathrm{L}$ $(0.025 \mathrm{IU} / \mathrm{mL})$ and $10 \mathrm{ng} / \mu \mathrm{L}(0.125 \mathrm{IU} / \mathrm{mL})$ from PBS and human serum with ratio 1:100 dilution

(a)

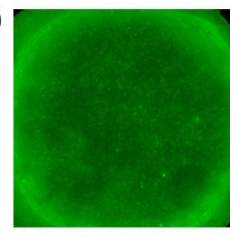

$100 \mathrm{ng} / \mu \mathrm{L}$

(b)

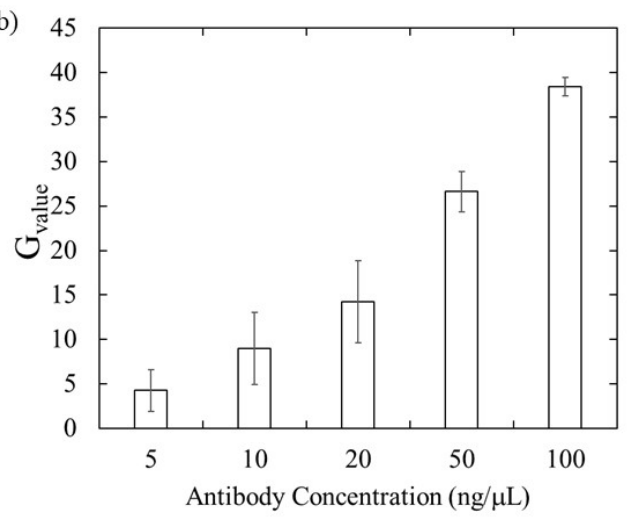

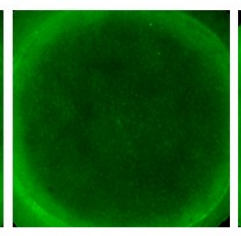

$10 \mathrm{ng} / \mu \mathrm{L}$

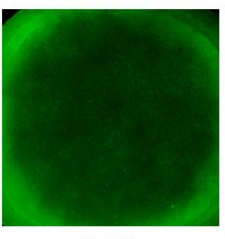

$5 \mathrm{ng} / \mu \mathrm{L}$

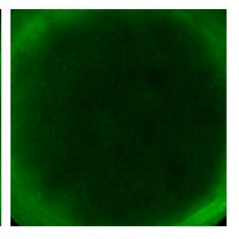

Negative Control

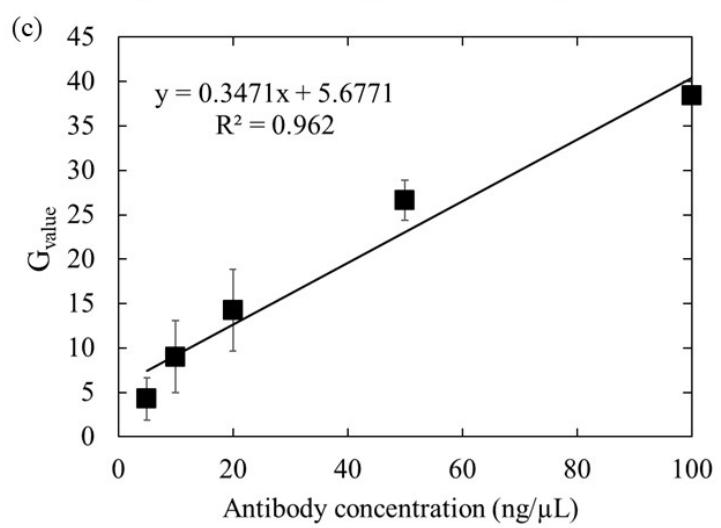

Figure 6 Sensitivity of the assay of SARS-CoV-2 humanized antibody spiked in human serum ratio of 1:100 (a) Fluorescent images of known SARS-CoV-2 humanized antibody concentration from 5 to 100 $\mathrm{ng} / \mu \mathrm{L}$ spiked in human serum ratio of 1:100 (b) The bar diagram for Gvalue observed from known SARS-CoV-2 humanized antibody concentration from 5 to $100 \mathrm{ng} / \mu \mathrm{L}$ spiked in human serum ratio of 1:100 (c) The relation of Gvalue and SARS-CoV-2 humanized antibody concentration spiked in human serum ratio of 1:100 and the linear calibration plot from 5 to $100 \mathrm{ng} / \mu \mathrm{L}$. 
Table 1 Material cost breakdown of paper-based fluorescent ELISA assay for SARS-CoV-2 humanized antibody detection

\begin{tabular}{ccc}
\hline Material list & Price/Quantity & Cost per 1000 assay \\
\hline Whatman Chromatography filter paper & $0.8-1 \$ / 20 \times 20 \mathrm{~cm}$ & $0.5-0.6 \$$ \\
Laminated film & $0.1-0.2 \$ / 48$ assay & $2.1-4.2 \$$ \\
Recombinant SARS-CoV-2 nucleocapsid & $700-800 \$ / 1 \mathrm{mg}$ & $1400-1600 \$$ \\
Rabbit Anti-human IgG & $140-160 \$ / 0.5 \mathrm{mg}$ & $8-10 \$$ \\
AlexaFluor-488 conjugation kits & $300-360 \$ / \mathrm{kit}$ & $600-720 \$$ \\
\hline Total & $2010-2335 \$$ \\
\hline Cost per assay & $2.01-2.34 \$ /$ Assay
\end{tabular}

respectively with \%RSD $\sim 6.4 \%$. While LOD and reproducibility of commercial ELISA kits in the market are $5 \mathrm{IU} / \mathrm{mL}$ with \%RSD 15-20\%. There are some distinct benefits in our assay. First, this assay can be completed within $15 \mathrm{~min}$, which is rapid than our previous study of colorimetric paper-based ELISA (30 min) and conventional ELISA (up to $1 \mathrm{~h}$ ).[18] Second, this assay can be used as an alternative serological test because SARS-CoV-2 antibody in human serum can be detected. However, sample preparation especially for human serum is still required because it causes a relatively high background signal which decreases the sensitivity of the assay. Third, the cost to perform an assay is around 2.01 - 2.34 USD (Table 1). We strongly believe that our assay has the potential to be a point-of-care diagnostic device which attains specificity, sensitivity, reproducibility, and suitability to work with more complex biological substance because our assay also produced promising data comparable to those of immunofluorescent serological assays. Nevertheless, pre-screening process with serological test for SARS-CoV-2 patients was more lagging than the symptom due to delay immune response.[24] Our paper-based assay, including serological test, could be used as a tool to confirm infected active cases or as a supporting assay to determine the infection stage as well as possible spread locations so that the health officials can control the spread more effectively.[25, 26] Furthermore, during vaccination trial period, our paper device could also contribute to evaluate vaccine efficiency in a large batch of human samples due to its portability, friendly-use and economical platform. [18]

Conflicts of interest: There are no conflicts to declare.

Acknowledgements: The authors acknowledge the support of the Australian Research Council (DP180100055) and higher degree research scholarships GUIPRS and GUPRS Scholarships to S.K. from the Griffith University.

\section{References}

[1] G. Caruana et al., "Diagnostic strategies for SARS-CoV-2 infection and interpretation of microbiological results," Clinical Microbiology and Infection.

[2] S. Mallapaty, "Where did COVID come from? Five mysteries that remain," Nature, vol. 591, pp. 188-189, 2021.

[3] F. Li, "Structure, Function, and Evolution of Coronavirus Spike Proteins," Annual Review of Virology, vol. 3, no. 1, pp. 237-261, 2016/09/29 2016.

[4] T. T. Tok and G. Tatar, "Structures and Functions of Coronavirus Proteins: Molecular Modeling of Viral Nucleoprotein," Int J Virol Infect Dis., vol. 2, no. 1, pp. 001-007, 2017.

[5] F. Wu et al., "A new coronavirus associated with human respiratory disease in China," Nature, vol. 579, no. 7798, pp. 265-269, 2020/03/01 2020.

[6] R. Kumar, S. Nagpal, S. Kaushik, and S. Mendiratta, "COVID-19 diagnostic approaches: different roads to the same destination," VirusDisease, vol. 31, no. 2, pp. 97-105, 2020/06/01 2020. 
[7] J. T. Wu, K. Leung, and G. M. Leung, "Nowcasting and forecasting the potential domestic and international spread of the 2019-nCoV outbreak originating in Wuhan, China: a modelling study," The Lancet, vol. 395, no. 10225, pp. 689-697, 2020/02/29/2020.

[8] D. P. Oran and E. J. Topol, "Prevalence of Asymptomatic SARS-CoV-2 Infection," Annals of Internal Medicine, 2020.

[9] B. J. Quilty et al., "The effect of travel restrictions on the geographical spread of COVID-19 between large cities in China: a modelling study," BMC Medicine, vol. 18, no. 1, p. 259, 2020/08/19 2020.

[10] K. Regmi and C. M. Lwin, "Impact of social distancing measures for preventing coronavirus disease 2019 [COVID-19]: A systematic review and meta-analysis protocol," medRxiv, p. 2020.06.13.20130294, 2020.

[11] J. Zhao et al., "Antibody responses to SARS-CoV-2 in patients of novel coronavirus disease 2019," Clinical Infectious Diseases, 2020.

[12] M. Wang et al., Clinical diagnosis of 8274 samples with 2019-novel coronavirus in Wuhan. 2020.

[13] R. T. Suhandynata, M. A. Hoffman, M. J. Kelner, R. W. McLawhon, S. L. Reed, and R. L. Fitzgerald, "Longitudinal Monitoring of SARS-CoV-2 IgM and IgG Seropositivity to Detect COVID-19," The Journal of Applied Laboratory Medicine, 2020.

[14] B. D. Kevadiya et al., "Diagnostics for SARS-CoV-2 infections," Nature Materials, 2021/02/15 2021.

[15] G. Liu and J. F. Rusling, "COVID-19 Antibody Tests and Their Limitations," ACS Sensors, vol. 6, no. 3, pp. 593-612, 2021/03/26 2021.

[16] M. Lipsitch, R. Kahn, and M. J. Mina, "Antibody testing will enhance the power and accuracy of COVID-19-prevention trials," Nature Medicine, vol. 26, no. 6, pp. 818-819, 2020/06/01 2020.

[17] C. W. Tan et al., "A SARS-CoV-2 surrogate virus neutralization test based on antibody-mediated blockage of ACE2-spike protein-protein interaction," Nature Biotechnology, 2020/07/23 2020.

[18] S. Kasetsirikul, M. Umer, N. Soda, K. R. Sreejith, M. J. A. Shiddiky, and N.-T. Nguyen, "Detection of the SARS-CoV-2 humanized antibody with paper-based ELISA," Analyst, 10.1039/D0AN01609H vol. 145, no. 23, pp. 7680-7686, 2020.

[19] C.-M. Cheng et al., "Paper-Based ELISA," Angewandte Chemie International Edition, vol. 49, no. 28, pp. 4771-4774, 2010/06/28 2010.

[20] Y. Jiao et al., "3D vertical-flow paper-based device for simultaneous detection of multiple cancer biomarkers by fluorescent immunoassay," Sensors and Actuators B: Chemical, vol. 306, p. 127239, 2020/03/01/2020.

[21] L. Guo et al., "Magnetic Quantum Dot Nanobead-Based Fluorescent Immunochromatographic Assay for the Highly Sensitive Detection of Aflatoxin B1 in Dark Soy Sauce," Analytical Chemistry, vol. 91, no. 7, pp. 4727-4734, 2019/04/02 2019.

[22] X. Qi, Y. Huang, Z. Lin, L. Xu, and H. Yu, "Dual-Quantum-Dots-Labeled Lateral Flow Strip Rapidly Quantifies Procalcitonin and C-reactive Protein," (in eng), Nanoscale research letters, vol. 11, no. 1, pp. 167-167, 2016.

[23] Thermo Fisher Scientific, Protein Assay Technical Handbook, 2017. [Online]. Available.

[24] L. J. Carter et al., "Assay Techniques and Test Development for COVID-19 Diagnosis," ACS Central Science, vol. 6, no. 5, pp. 591-605, 2020/05/27 2020.

[25] L. Fernández-Barat, R. López-Aladid, and A. Torres, "The value of serology testing to manage SARS-CoV-2 infections," European Respiratory Journal, vol. 56, no. 2, p. 2002411, 2020. 
[26] R. W. Peeling et al., "Serology testing in the COVID-19 pandemic response," The Lancet Infectious Diseases, vol. 20, no. 9, pp. e245-e249, 2020. 